\title{
THE NEW OPACITIES AND B-STAR PULSATIONS
}

\author{
W.A. DZIEMBOWSKI \\ Nicolaus Copernicus Astronomical Center, Bartycka 18, 00-716 Warsaw, Poland
}

\begin{abstract}
Over thirty years ago Baker \& Kippenhahn (1962) demonstrated that an instability driven by the opacity mechanism is the cause of Cepheid pulsations. Recently it has been shown that the same mechanism is responsible for oscillations observed in $\beta$ Cephei, SPB and perhaps in other variable B-type stars. The search for the driving mechanism in hot stars began in the late sixties with no success until the opacities calculated with the OPAL code (Iglesias, Rogers and Wilson 1990, 1992) became available. The crucial new feature in the opacity is the local maximum at $T \approx 2 \times 10^{5} \mathrm{~K}$ caused by iron lines which was ignored in earlier calculations. Recently, stellar opacity data from an independent project (OP) became available (Seaton et al., 1993). The agreement between the two opacity data is satisfactory.

In $B$ stars the opacity mechanism drives two distinct categories of normal modes. The one relevant to $\beta$ Cep stars encompasses low order $p$ - and $g$-modes with periods $0.1-0.3 \mathrm{~d}$. The other includes high-order $g$-modes with periods ranging up above $4 \mathrm{~d}$. Excitation of such modes may explain most of the slow variability observed in $B$ stars. The theoretical instability domain in the $\mathrm{H}-\mathrm{R}$ diagram is very sensitive to metal abundance. For the standard value of $Z=0.02$, the total instability domain in the main sequence band extends from spectral type 09.5 to B9. In types later than B2 only high-order $g$-modes are unstable.
\end{abstract}

\section{The Opacity Mechanism}

For many years, explaining the cause of $\beta$ Cephei variability has been a major challenge to stellar pulsation theory. The problem is now solved, but we owe the solution to progress in opacity calculations and not to new astrophysical ideas. In fact, if the OPAL opacities were available the problem would have been solved many years ago (Baker \& Dziembowski 1969, unpublished).

What drives pulsation in $\beta$ Cep stars is the opacity mechanism-the same as in classical pulsating variables-except that it relies on a different opacity bump. The spontaneous excitation of an oscillation mode is caused by a particular behaviour of the opacity. In the high-pressure phase of oscillation, the Rosseland mean opacity, $\kappa$, increases in the outer layers. This causes a capturing of part of the radiative flux and converting its energy into pulsation energy. The net energy gain during one cycle, $W$, usually called the work integral, is given by

$$
W=-\int d^{3} \mathbf{x} \nabla_{\mathrm{ad}} \oint d t \frac{\delta p}{p} \delta \nabla \cdot \mathbf{F},
$$

where $\delta$ denotes the Lagrangian perturbation, $p$ is the gas+radiation pressure, $\mathbf{F}$ is the radiative flux and $\nabla_{\mathrm{ad}}=\left(\frac{d \ln p}{d \ln T}\right)_{\mathrm{ad}}$. The expression is valid if the amplitude growth rate is much longer than the period. However, locally the departure from adiabaticity may be arbitrarily large. Furthermore, it applies 
both to radial and nonradial perturbations. The subsequent formulae are strictly valid only for radial perturbation. They remain approximately valid for all nonradial modes considered in this review, but we have to remember that there is an implicit spherical harmonic factor in the perturbed quantities. We have

$$
\delta \nabla \cdot \mathbf{F}=\frac{1}{4 \pi r^{2}} \frac{d \delta L_{r}}{d r},
$$

where $L_{r}$ is the local luminosity, and

$$
\frac{\delta L_{r}}{L}=\frac{d r}{d \ln T} \frac{d}{d r} \frac{\delta T}{T}-\frac{\delta \kappa}{\kappa}+4\left(\frac{\delta T}{T}+\frac{\delta r}{r}\right) .
$$

The opacity mechanism may work only if the second term is large. Depending on the form of the $\kappa(T, \rho)$-dependence, this term may contribute either to a mode excitation or to its damping. In ordinary terrestrial conditions the first term dominates and its net effect is always a wave damping.

The plots shown in Fig. 1 will help to remind us how the opacity mechanism works in Cepheids. We see in the bottom panel that the dominant positive contribution to driving arises in a narrow zone around $\log T \approx 4.65$. In this zone $\delta L_{r}$ decreases outwards, while both $\delta \kappa / \kappa$ and $\kappa_{T}$ increase. The direction of change is opposite in the zone around $\log T \approx 4.85$ where most of the negative contribution to $W$ arises. Let us note, parenthetically, that simplified analytical models such as Baker's one-zone model assuming constant $\kappa_{T}$, miss the most important aspect of the opacity mechanism.

The local maximum of $\kappa_{T}$ at $\log T \approx 4.75$ is caused by He II ionization. A bigger maximum at $\log T \approx 4.1$ occurring in the $\mathrm{H}$ ionization zone is much less significant for the mode stability despite the fact that the eigenfunctions $\delta T / T$ and $\delta \kappa / \kappa$ are big and rapidly varying. This is because the thermal time scale of the $\mathrm{H}$ ionization zone is much shorter than the pulsation period and therefore the tendency towards thermal equilibrium prevails, which enforces $\boldsymbol{\nabla} \cdot \mathbf{F} \approx 0$. The third maximum located at $\log T \approx 5.3$ is the new opacity feature causing instability in $\beta$ Cep models. The contribution to $W$ from this deep layer is very small because the $\delta T / T$ amplitude is very small there.

The occurrence of a local maximum of $\kappa_{T}$ at the proper location within the stellar model is the condition for mode excitation through the opacity mechanism. There are corresponding maxima in the opacity itself occurring at somewhat higher temperatures. We will refer to the three opacity maxima seen in Fig. 1 as the H, the He II and the metal bump. In different models, the three bumps occur at approximately the same temperature as in the model used in Fig. 1, but at different geometrical depths. The He II bump is the primary cause of pulsations in classical Cepheids, W Vir, RV Tau, RR Lyr and $\delta$ Sct stars. This has been convincingly demonstrated in a large number of papers following the pioneering work of Baker \& Kippenhahn (1962). In cooler stars, the $\mathrm{H}$ bump is located in deeper layers and consequently it 


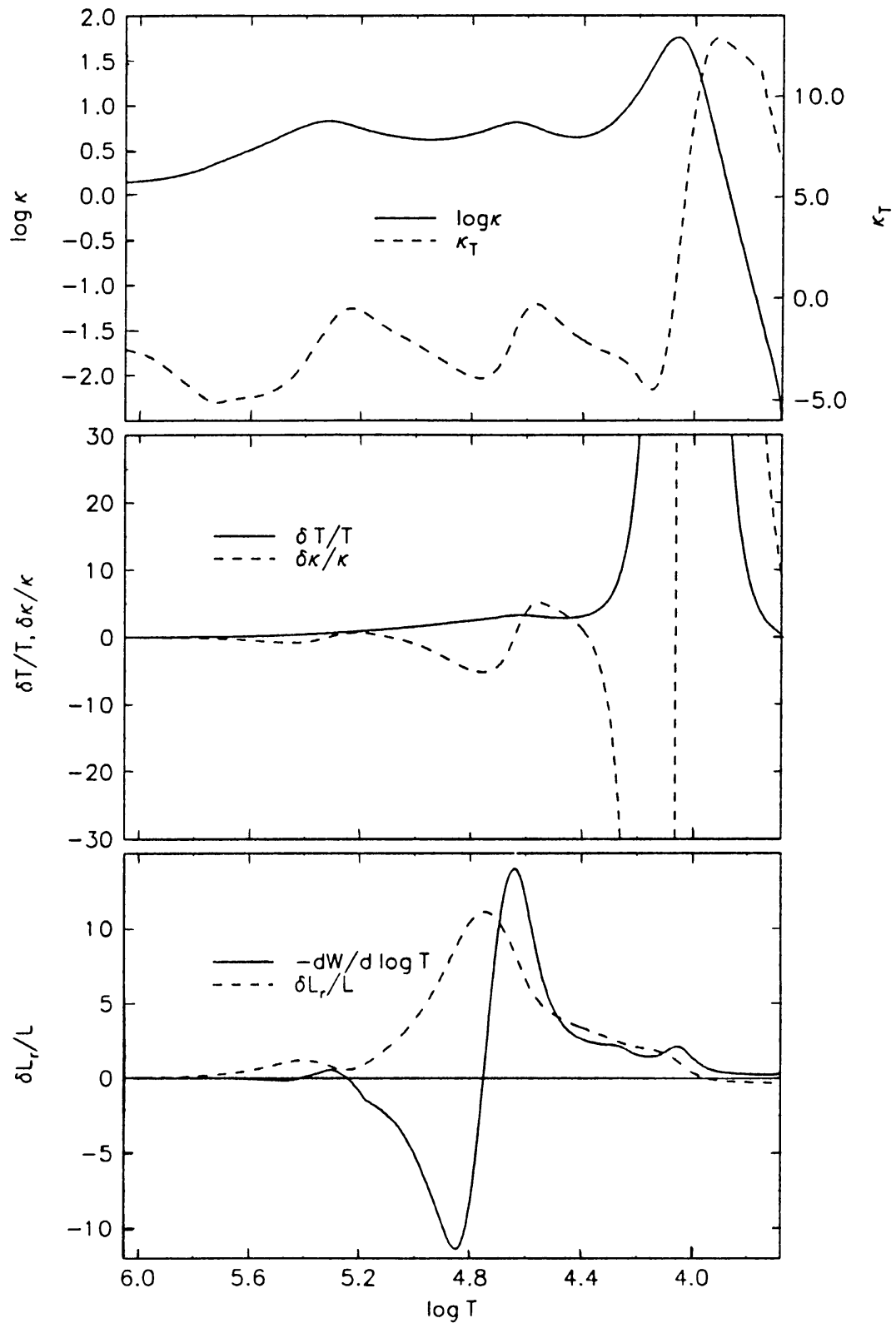

Fig. 1. Opacity $\kappa$, opacity derivative $\kappa_{T}=\left(\frac{\partial \ln \kappa}{\partial \ln T}\right)_{\rho}$, eigenfunctions describing relative changes of selected parameters (temperature $\frac{\delta T}{T}$, opacity $\frac{\delta \kappa}{\kappa}$, local luminosity $\frac{\delta L_{x}}{L}$ ) and the differential work integral $d W / d \log T$ (arbitrary units, positive in driving zones) in the envelope of a $\delta$ Cep star model $\left(M=6 M_{\odot}, \log \left(L / L_{\odot}\right)=3.32, \log T_{\text {eff }}=3.75\right)$. Pulsation characteristics refer to the fundamental radial mode (period 5.54 day) and to the phase of maximum pressure. The abscissa value $\log T=6$ corresponds to the fractional radius value $r / R=0.26$. 
may play a dominant role in driving pulsations in cooler stars. In several studies, the critical role of the $\mathrm{H}$ bump in Mira models has been confirmed. In oscillating DA and DB White Dwarfs the excitation takes place in the $\mathrm{H}$ and He II ionization zones, respectively. However, because of the critical role of convective transport in these cases, the driving effect cannot be regarded as the classical opacity mechanism.

\section{The OPAL and OP Opacities}

In the Los Alamos opacities, which were commonly used for stellar structure calculations until 1991 , there is no bump for $\log T>4.8$. At $\log T \approx 5.3$ and the corresponding density, the $\kappa$ value is nearly a factor three less than in the new opacities used in Fig. 1. How was it possible that such a big effect was overlooked? Why was it that even after Simon (1982) had pointed out that an opacity bump located at $\log T \approx 5.3$ would help to resolve the two outstanding problems in stellar pulsations, the Los Alamos team (Magee, Merts \& Hubner, 1984) decidedly dismissed such a possibility? It was perhaps because intuition suggests to us that strong lines are important and weak lines are an unimportant. However, since the $\kappa$ coefficient is the harmonic mean of monochromatic opacities, the truth may be the exact opposite.

The opacity calculations are complicated, and the accuracy requirements are high. It is therefore very fortunate that the task of re-examination of stellar opacities was undertaken by two independent groups. The Lawrence Livermore National Laboratory group developed a sophisticated opacity code named OPAL. The other was a large international group involved in the "Opacity Project" (OP). I use the names OPAL and OP to denote values of the Rosseland mean opacity published by the two respective groups.

The existence of a large bump in the Rosseland mean opacities for temperatures near $2 \times 10^{5} \mathrm{~K}$, caused mostly by iron lines, was already demonstrated in an early version of the OPAL opacities (Iglesias, Rogers \& Wilson 1990, Rogers \& Iglesias 1992). This qualitatively new feature was found thanks to an improved treatment of atomic transitions which specifically included term structures in electron configurations. The most recent version of the opacities (Iglesias, Rogers, \& Wilson 1992) takes into account spin-orbit interactions for the heavier elements and results in a further enhancement of the bump. An early version of the OPAL opacities has already established the instability of certain modes in B-star models (Cox et al. 1992; Kiriakidis, El Eid \& Glatzel 1992; Moskalik \& Dziembowski 1992). However, only calculations employing the improved version (Dziembowski \& Pamyatnykh 1993; Gautschy \& Saio 1993) explain the occurrence of the $\beta$ Cep domain in the $\mathrm{H}-\mathrm{R}$ diagram without invoking abnormally high metal abundances.

The OP opacities became available only very recently (Seaton et al. 1993). 

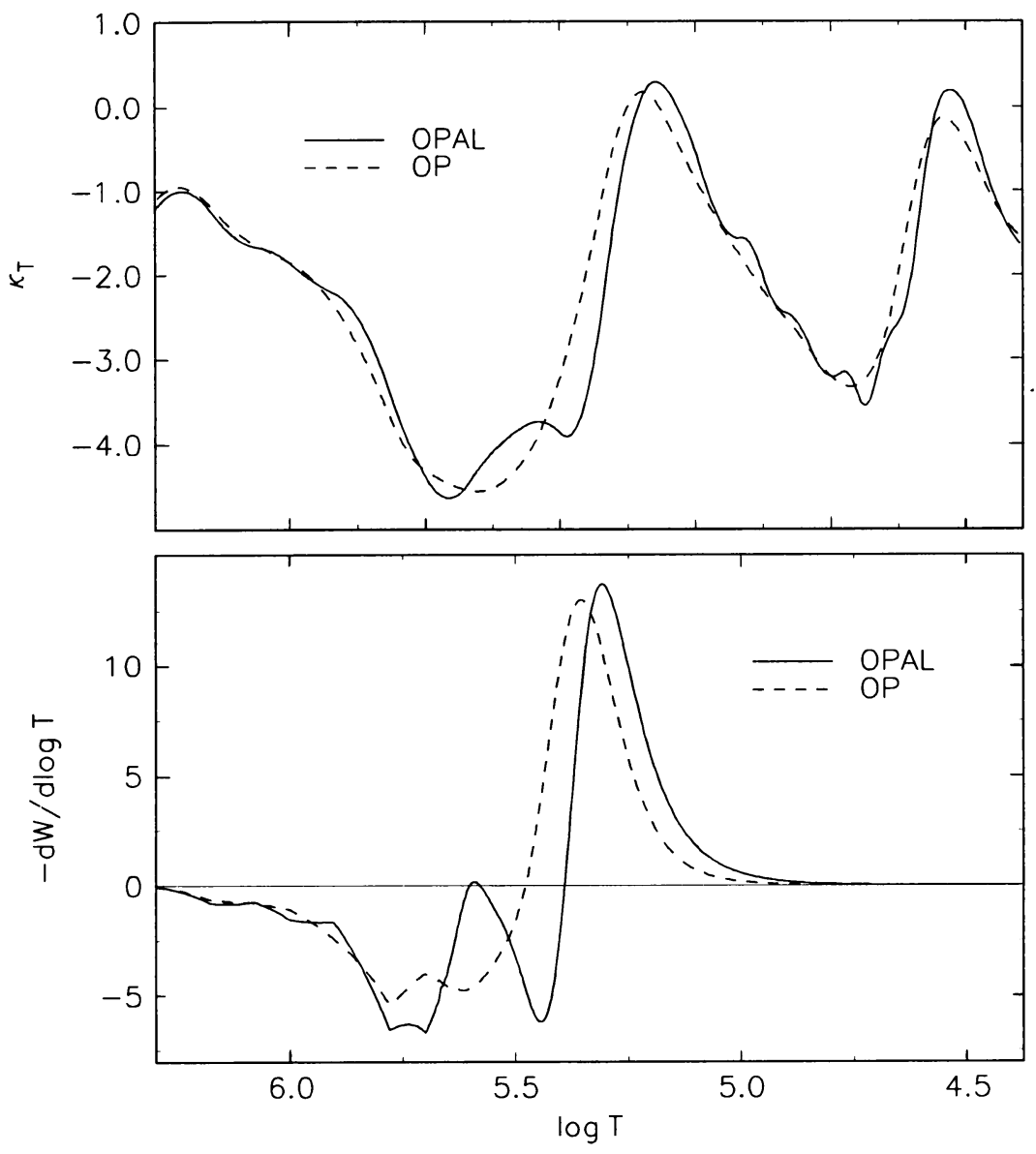

Fig. 2. The opacity derivative $\kappa_{T}$, and differential work integral $-d W / d \log T$ for the fundamental radial mode in the envelope of a $\beta$ Cep star model $\left(M=12 M_{\odot}\right.$, $\left.\log \left(L / L_{\odot}\right)=4.40, \log T_{\text {eff }}=4.24\right)$ calculated with two different opacity data. The maximum abscissa value of $\log T=6.29$ corresponds to the fractional radius value $r / R=0.71$. The $p_{0}, l=0$ mode is unstable only in the model calculated with OPAL opacities.

The approach adopted in this project was different. In particular, plasma effects were ignored in calculating atomic radiative properties. This leads to a considerable simplification in computation and allows a more accurate treatment of electron transitions. In the case of B-star envelopes calculated with the same chemical composition, the agreement between the results obtained with the two opacities is very encouraging. The comparison was done with a test version of OP calculated for the composition adopted in the OPAL opacities. In the standard version, the three iron-group elements $(\mathrm{Cr}$, $\mathrm{Mn}$ and $\mathrm{Ni}$ ) are added and this leads to a significant improvement relative to the OPAL opacities. 
The important difference between the two opacities consists of a slight displacement of the metal bump towards higher temperatures. This is shown in Fig. 2, where the critical opacity derivative in a $\beta$ Cep star model is plotted. In the same figure we plot the differential work integral for the fundamental radial mode. In this case the bump displacement causes mode destabilization. A more general consequence of switching from the OPAL to OP opacity data is a displacement of the instability domain in the H-R diagram toward higher $T_{\text {eff }}$ and $L$, which is a straightforward consequence of the bump displacement. More details on comparison of results obtained with the two opacities are given in the contribution by Pamyatnykh et al. (these Proceedings).

\section{Unstable Oscillation Modes}

In models of $\beta$ Cep type stars with the standard value of the metal abundance parameter $Z=0.02$, the instability of radial modes is restricted to the fundamental mode and the first overtone. The latter is unstable only for stellar masses $M \geq 10 M_{\odot}$. Simultaneously, there is always a large number of unstable nonradial modes. They come in two distinct classes. The first class, characterized by periods similar to those of unstable radial modes, encompasses low-order $\boldsymbol{p}$ - and $\boldsymbol{g}$-modes. The second class, present only at sufficiently high $l$ - values, encompasses a range of high-order $g$-modes with significantly longer periods.

As an illustration, we consider the $12 M_{\odot}$ model calculated with the OP opacities used in the previous section. In this model we have two unstable modes $\left(p_{1}\right.$ and $\left.p_{2}\right)$ for $l=0$. The $g_{1}$ and $p_{1}$ modes are unstable for $l=1$. All these modes have periods in the range $0.146-0.192 \mathrm{~d}$, which is typical for $\beta$ Cep stars. At $l=8$ we have one unstable mode in this period range. The mode may be classified as $p_{0}$, because most of its kinetic energy comes from the acoustic cavity where its radial displacement eigenfunctions have no node, or as $g_{2}$ because there are two nodes in the gravity-wave cavity close to the boundary of the convective core. Modes from $g_{3}-g_{14}$ are stable and those from $g_{14}-g_{25}$ are again unstable. The second instability range corresponds to the $0.42-0.76 \mathrm{~d}$ period range. Plots of the pressure eigenfunction and the differential work integral for three selected modes as shown in Fig. 3 will help us understand the origin of the two instability ranges. The crucial aspect is the shape of the pressure eigenfunction. For both $p_{0}$ and $g_{21}$ and unlike $g_{10}$, the amplitude is large only in the outer layers where driving occurs. The amplitude behaviour in the interior $(\log T>6)$ for the two former modes is vastly different, but the contribution to $W$ from these layers is negligible in both cases.

At lower $l$-values the modes from $g_{14}$ to $g_{25}$ have an eigenfunction behaviour similar to those at $l=8$, and thus favorable for excitation. However, their 

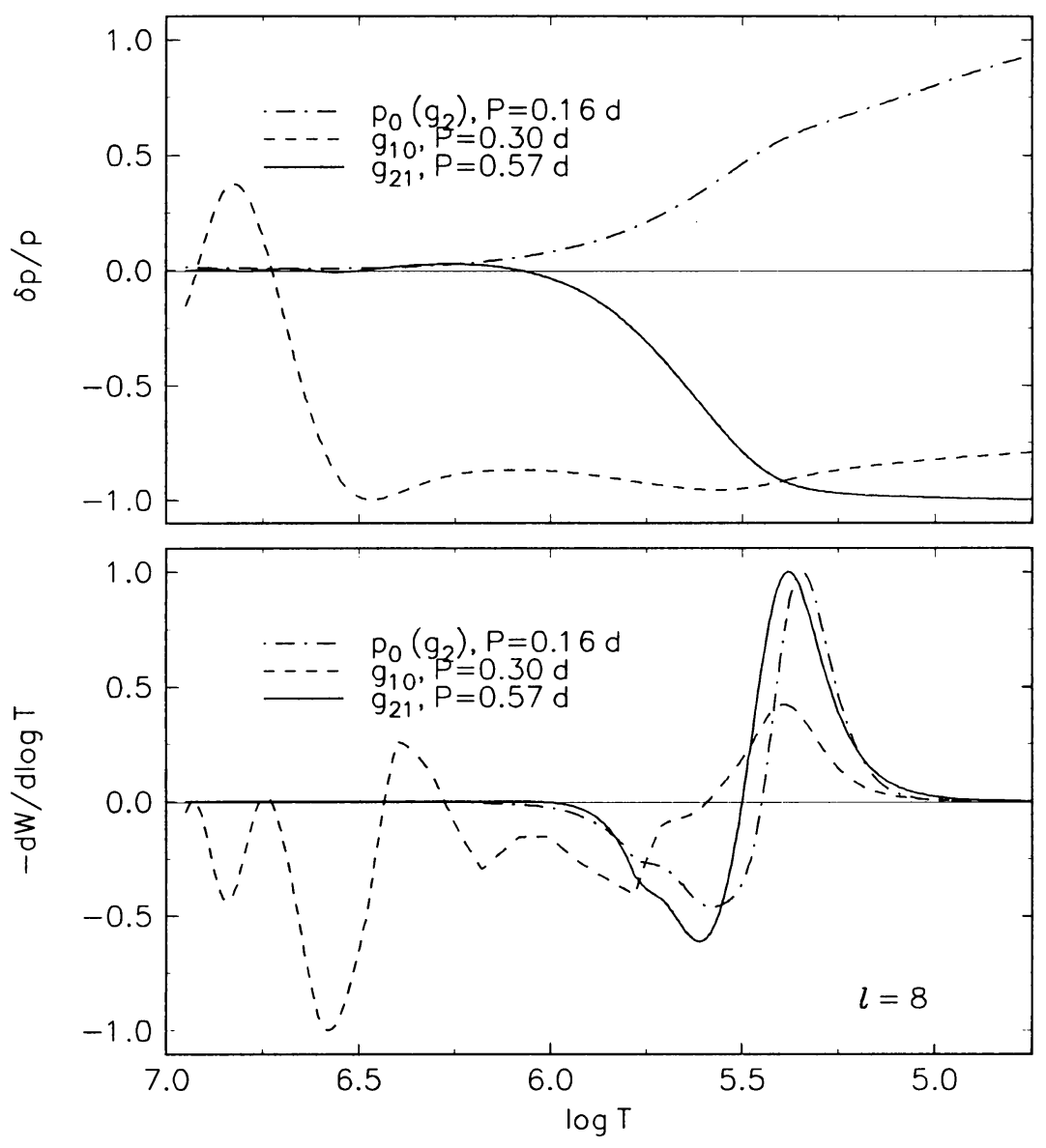

Fig. 3. The radial dependence for the $\delta p / p$ eigenfunction and the differential work integral for selected $l=8$ modes but in a different $\log T$-range, corresponding to the $r / R$-range $0.35-0.993$. The model is the same as used in Fig. 2.

periods, which at fixed radial order are proportional to $\sqrt{l(l+1)}$, are too long for efficient driving in the metal bump region. The low- $l$, high-order $g$-modes are unstable in less luminous star models, because in such stars the metal bump is located in deeper layers and consequently its thermal time scale is longer. Excitation of such modes has been proposed (Dziembowski \& Pamyatnykh 1993; Gautschy \& Saio 1993; Dziembowski, Moskalik \& Pamyatnykh 1993) as an explanation of the slow variability observed in mid and late B-type stars. 


\section{The B-star Instability Strip}

The instability domains in the H-R diagram are shown in Fig. 4. The plots are based on a stability survey (Pamyatnykh et al., in preparation), for low-l modes. Stellar models were calculated with the OP opacities for the abundance parameter values $X=0.7$ and $Z=0.02$.

Only high-order $g$-modes are unstable in the lower domain. The periods of the unstable modes range from 0.5 to above $4 \mathrm{~d}$. This range is consistent with periodicities observed in variable objects lying in this part of the H-R diagram. The name SPB stars was used after Waelkens (1991) who discovered most of these objects and who demonstrated that they are $g$-mode pulsators. The instability of high-order $g$-modes is present in more luminous and hotter stars but only at higher degrees, $l$. It is possible that the spectral line changes observed in some B (mainly Be) stars are caused by the excitation of such modes. The role of $g$-modes in Be stars should be clarified not only in the variability context, but also as a possible cause of their activity.

The $\beta$ Cep stars occur only in a small part of the B-star instability strip. Their observational domain in the H-R diagram is limited to the advanced main-sequence phase of 9-18 $M_{\odot}$ stars. The lack of objects in the post-MS phase is naturally explained by the speed of evolution. There is a paucity of pulsating stars near the ZAMS predicted by theory in the mass range 8-10 $M_{\odot}$. I do not know whether this reflects an inadequacy in the linear stability calculations or the difference in the nonlinear behaviour between low- and high-mass stars.

The B-star instability strip widens into the supergiant region and may even merge with the extension of the $\delta$ Cep instability strip (Zalewski, in preparation). Thus it seems possible that the widespread variability of extreme supergiants may also be explained by the opacity mechanism.

\section{Prospects and Problems}

Identification of the instability mechanism of $\beta$ Cep stars does not eliminate these stars from the list of interesting astronomical objects. The solution of the puzzle of their pulsations, which may not seem very interesting, certainly has a bright side. We can now extract much more interesting information about these stars and the systems they live in from the observational data. The occurrence of $\beta$ Cep stars in a stellar system may now be used to infer information about age and chemical composition.

Having credible linear nonadiabatic models for oscillations in these stars, we may use the information contained in the amplitude ratios light/colour and light/(radial velocity) and the corresponding phase differences to infer the stellar parameters (Cugier et al., these Proceedings). These data in combination with the frequencies should be regarded as valuable asteroseismo- 


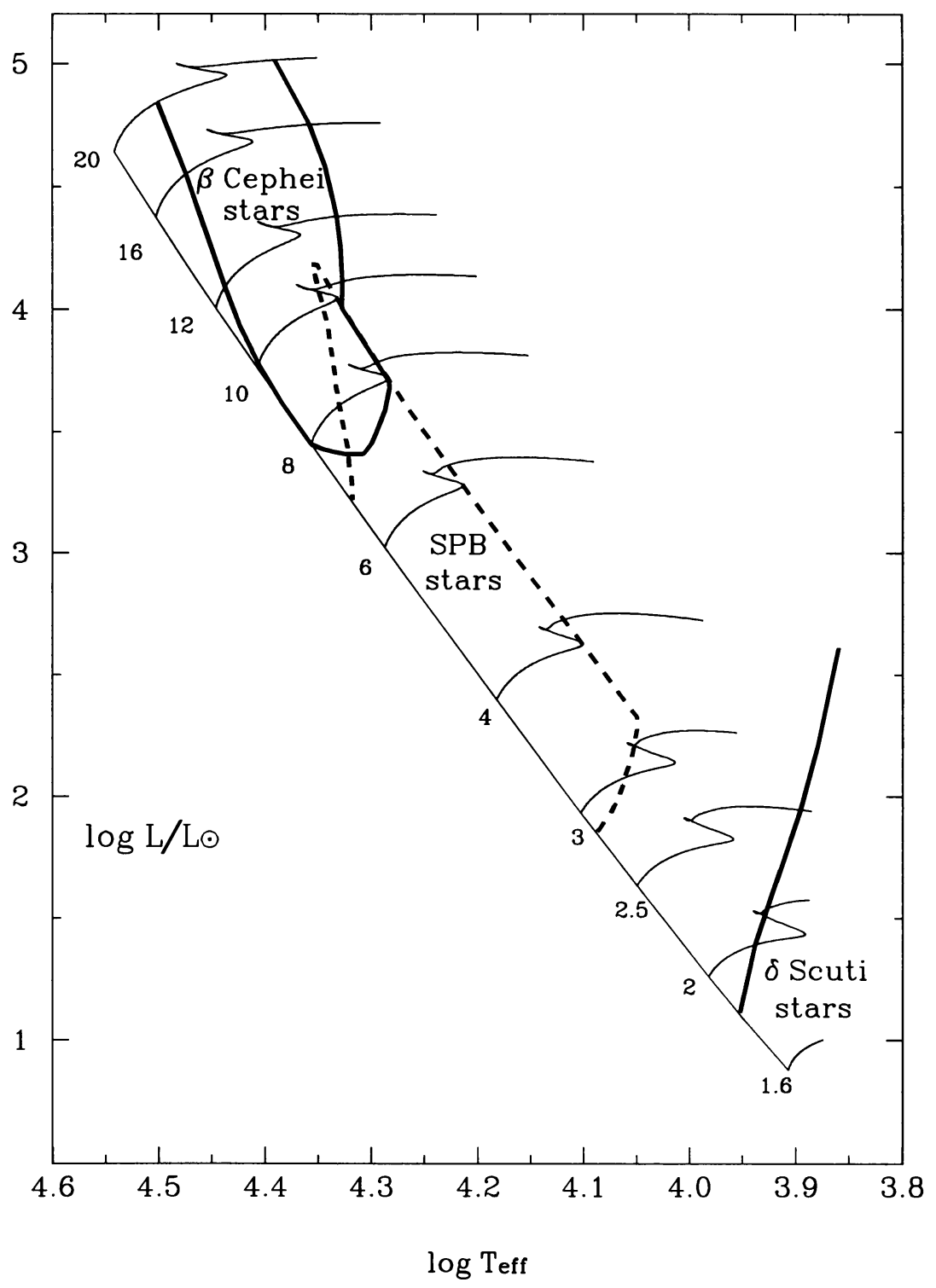

Fig. 4. The boundaries of the two domains in the H-R diagram where low-l mode are unstable due to the driving effect of the metal opacity bump. Within the boundaries there are models having at least one unstable mode with $l \leq 2$. In the upper ( $\beta$ Cep) domain the unstable modes are low-order $p$ - and/or $g$-modes and in the lower (SPB) domain the unstable modes are high-order $g$-modes. The blue edge of the low luminosity extension of the Cepheid instability strip is also shown ( $\delta$ Sct stars). 
logical information. Numerous multimodal objects are particularly attractive targets. The same applies to SPB stars, though their long periods make these objects more difficult for observation. It is important that in the case of B-type pulsators, the predictions of stellar pulsation theory are not affected by the lack of a satisfactory description of convective transport. A thin convective layer does appear in the driving zone of more luminous B stars, but the estimated convective flux is far less significant than in the case of Cepheid-type pulsators.

Our understanding of B star oscillations is still not satisfactory. We know almost nothing about what happens at finite amplitudes. Nonlinear studies of $\beta$ Cep models started only very recently (Moskalik \& Buchler, these Proceedings). The linear theory predicts instability of many modes. We do not know which of them will be present in the nonlinear development and what their amplitudes will be or whether the amplitudes will be constant or varying in time. If the latter is true, we do not know whether the variability will be periodic or chaotic. We do not even know whether for a specified stellar model there is a unique answer to these questions. The problems are not specific to B- type pulsators, but because convection is least significant in these stars, these are perhaps the best objects for exploring complex nonlinearities in stellar pulsation theory.

One of the biggest puzzles is the origin of activity in Be stars. The possibility that the excitation of $g$-modes plays a role remains to be explored. A necessary first step which is still within the framework of the linear theory, is to include effects of rotation in the stability calculations.

\section{Acknowledgements}

I am grateful to Alosha Pamyatnykh for Fig. 4 and many very helpful conversations. The assistance of the LOC and IAU in financing my participation in the Symposium is gratefully acknowledged.

\section{References}

Baker, N. and Kippenhahn, R.: 1962, Zeit. Astrophys. 54, 114.

Cox, A.N., Morgan, S.M., Rogers, F.J. and Iglesias, C.A.: 1992, Astrophys. J. 393, 272.

Dziembowski, W.A., Moskalik, P. and Pamyatnykh, A.A.: 1993, Mon. Not. Roy. Astr. Soc. in press.

Dziembowski, W.A. and Pamyatnykh, A.A.: 1993, Mon. Not. Roy. Astr. Soc. 262, 204.

Gautschy, A. and Saio, H.: 1993, Mon. Not. Roy. Astr. Soc. 262, 213.

Iglesias, C.A., Rogers, F.J. and Wilson, B.G.: 1990, Astrophys. J. 360, 221.

Iglesias, C.A., Rogers, F.J. and Wilson, B.G.: 1992, Astrophys. J. 397, 717.

Kiriakidis, M., El Eid, M.F. and Glatzel, W.: 1992, Mon. Not. Roy. Astr. Soc. 255, 1p.

Magee, N.H., Merts, A.L. and Huebner, W.F.: 1984, Astrophys. J. 283, 264.

Moskalik, P. and Dziembowski, W.A.: 1992, Astron. Astrophys. 256, L5.

Rogers, F.J. and Iglesias, C.A.: 1992, Astrophys. J. 79, 507. 
Seaton, M.J., Yu Yan, Mihalas, D. and Pradhan, A.K.: 1993, Mon. Not. Roy. Astr. Soc. in press.

Simon, N.R.: 1982, Astrophys. J. Let. 260, 87.

Waelkens, C.: 1991, Astron. Astrophys. 246, 453.

\section{Discussion}

Percy: Could you comment on the pulsation of B supergiants? Which modes (periods) are excited?

Dziembowski: So far, Zalewski (in preparation) has considered only radial modes. In addition to the fundamental mode and first overtone, he found unstable strange modes i.e. modes which have no counterpart in the adiabatic approximation. Their periods are always shorter than the fundamental mode period. These modes are responsible for merging the high-luminosity extension of the Cepheid and B-star instability strips.

Balona: The new opacities clearly show that there exists a region of instability for non-rotating mid-B stars. Recent observations of mine of two open clusters, NGC 3293 and NGC 4755 have failed to find any pulsating stars in the predicted strip. One may speculate that the relatively high rotational velocity in the cluster stars may be damping the pulsations. Can you say anything about the role of rotation in these stars?

Dziembowski: Our stability surveys were done ignoring effects of rotation. The results are not directly applicable to the case when the $g$-mode frequency is comparable with the rotation frequency. When the equatorial velocity exceeds some $50 \mathrm{~km} \mathrm{~s}^{-1}$ the properties of low- $l$ high-order $g$-modes are significantly changed by rotation. I cannot say whether such modes will be stabilized.

Waelkens: It is a significant observational fact that SPBs are slow rotators. All stars for which I found multiperiodic photometric variations had been selected on photometric grounds only, but turned out to be narrow-lined, with $v \sin i$ values of the order of $30 \mathrm{~km} \mathrm{~s}^{-1}$ or lower. Apparently, pulsations are suppressed in rapid rotators, or they have such large $l$-values that they are not seen in the photometry.

Henrichs: What do you mean by your conclusion that the origin of activity in Be stars might be understood?

Dziembowski: We have established that in all B stars, there is a mechanism converting the radiative flux energy directly into mechanical energy. It is a crucial step and, admittedly, the easiest, on the road to explaining the activity. The next step lies in the domain of the nonlinear theory. One may imagine, for instance, dynamo action of the $g$-modes. An asymmetry between prograde and retrograde modes, which is expected if rotation effects are significant, would lead to a net field amplification. 
Henrichs: Do you expect an instability strip in the Magellanic Clouds to occur with the new opacities?

Dziembowski: We (Dziembowski, Moskalik \& Pamyatnykh 1993) checked that with the metal abundance parameter $Z=0.01$, which is above the current upper limit for the SMC and perhaps also for the LMC. The $\beta$ Cep domain disappears, while the SPB domain is still present. We are currently working on precise criteria for both types of instability.

Kjeldsen: Concerning short periodic pulsators ( $\beta$ Cep stars) in the LMC and SMC, I want to point out that we (Kjeldsen \& Baade, these Proceedings) have possibly detected two $\beta$ Cep stars in the open cluster NGC 371 in the SMC. The amplitudes are $2-3 \mathrm{mmag}(P \approx 2 \mathrm{~h})$ and if these pulsations are real, it shows that even in environments with low $[\mathrm{Fe} / \mathrm{H}]$ the $\kappa$-mechanism is working.

Dziembowski: This is a very interesting result, but if confirmed I would be more inclined to suspect that the environment is not as low $[\mathrm{Fe} / \mathrm{H}]$ as we think.

Jerzykiewicz: You get a large number of $g$-modes excited in models of Btype stars. But your theory is linear, while we in fact observe real, non-linear objects. What would be the number of $g$-modes in the nonlinear case?

Dziembowski: At this stage I can say only that it will be greater than zero. However, this does not mean that an object lying within the instability strip must show detectable variability. One may imagine that the driving mechanism is saturated with one or more high-l modes, which even at large intrinsic amplitudes will not be seen. 